\title{
Erratum to: Testing Calendar Effects of International Equity and Real Estate Markets
}

\author{
Eddie C. M. Hui ${ }^{1}$ - Ka Kwan Kevin Chan ${ }^{1}$
}

Published online: 31 August 2017

(C) Springer Science+Business Media, LLC 2017

\section{Erratum to: J Real Estate Finan Econ DOI 10.1007/s11146-016-9564-1}

Unfortunately, there was an error in Table 4 in the original publication of the article. The correct version of the table is given below.

1 Department of Building and Real Estate, The Hong Kong Polytechnic University, Hong Kong, Hong Kong 
Table 4 The test results for the overall effect using our method

HSI
\begin{tabular}{|r|c|c|c|c|c|c|c|c|c|c|c|c|c|}
\hline Moving-window size & Jan & Feb & Mar & Apr & May & Jun & Jul & Aug & Sep & Oct & Nov & Dec & $95 \%$ confidence interval \\
\hline 40 & 0.554502 & 0.463542 & 0.399522 & 0.459658 & 0.610451 & 0.48642 & 0.591017 & 0.661098 & 0.587224 & 0.531915 & 0.659259 & 0.64455 & $(0.4895,0.6202)$ \\
\hline 80 & 0.732227 & 0.598958 & 0.492823 & 0.442543 & 0.472684 & 0.4 & 0.477541 & 0.548926 & 0.641278 & 0.595745 & 0.602469 & 0.630332 & $(0.4879,0.6181)$ \\
\hline 120 & 0.658768 & 0.625 & 0.638756 & 0.594132 & 0.482185 & 0.451852 & 0.489362 & 0.4812 & 0.616078 & 0.617021 & 0.696296 & 0.661137 & $(0.5193,0.6489)$ \\
\hline 160 & 0.687204 & 0.65625 & 0.62201 & 0.674817 & 0.605701 & 0.550617 & 0.51773 & 0.563246 & 0.53317 & 0.56974 & 0.632099 & 0.632701 & $(0.5387,0.66683)$ \\
\hline 200 & 0.637441 & 0.625 & 0.641148 & 0.669927 & 0.617577 & 0.585185 & 0.65721 & 0.646778 & 0.660934 & 0.588652 & 0.587654 & 0.623223 & $(0.5642,0.6928)$ \\
\hline 240 & 0.540284 & 0.585938 & 0.578947 & 0.621027 & 0.653207 & 0.644444 & 0.593381 & 0.675418 & 0.685504 & 0.640662 & 0.612346 & 0.616114 & $(0.5562,0.68551)$ \\
\hline
\end{tabular}

TPX
\begin{tabular}{|r|c|c|c|c|c|c|c|c|c|c|c|c|c|}
\hline Moving-window size & Jan & Feb & Mar & Apr & May & Jun & Jul & Aug & Sep & Oct & Nov & Dec & $95 \%$ confidence interval \\
\hline 40 & 0.597156 & 0.533854 & 0.526316 & 0.572127 & 0.565321 & 0.444444 & 0.567376 & 0.412888 & 0.390663 & 0.401891 & 0.409877 & 0.476303 & $(0.4258,0.5576)$ \\
\hline 80 & 0.518957 & 0.554688 & 0.631579 & 0.594132 & 0.584323 & 0.575309 & 0.562648 & 0.315036 & 0.353808 & 0.283688 & 0.360494 & 0.395735 & $(0.4124,0.5416)$ \\
\hline 120 & 0.490521 & 0.526042 & 0.607656 & 0.599022 & 0.562945 & 0.580247 & 0.574468 & 0.431981 & 0.383292 & 0.286052 & 0.325926 & 0.42654 & $(0.4176,0.5477)$ \\
\hline 160 & 0.473934 & 0.455729 & 0.5 & 0.577017 & 0.510689 & 0.533333 & 0.579196 & 0.448687 & 0.425061 & 0.380615 & 0.291358 & 0.374408 & $(0.3971,0.5282)$ \\
\hline 200 & 0.362559 & 0.408854 & 0.552632 & 0.459658 & 0.453682 & 0.474074 & 0.576832 & 0.534606 & 0.481572 & 0.347518 & 0.365432 & 0.334123 & $(0.3808,0.5115)$ \\
\hline 240 & 0.485782 & 0.5 & 0.397129 & 0.435208 & 0.444181 & 0.451852 & 0.484634 & 0.463007 & 0.452088 & 0.484634 & 0.414815 & 0.42891 & $(0.3872,0.5196)$ \\
\hline
\end{tabular}

SPX
\begin{tabular}{|r|c|c|c|c|c|c|c|c|c|c|c|c|c|}
\hline Moving-window size & Jan & Feb & Mar & Apr & May & Jun & Jul & Aug & Sep & Oct & Nov & Dec & $95 \%$ confidence interval \\
\hline 40 & 0.729858 & 0.588542 & 0.600478 & 0.665037 & 0.665083 & 0.540741 & 0.586288 & 0.470167 & 0.552856 & 0.579196 & 0.720988 & 0.755924 & $(0.5579,0.6853)$ \\
\hline 80 & 0.800948 & 0.734375 & 0.717703 & 0.660147 & 0.684086 & 0.590123 & 0.605201 & 0.491647 & 0.587224 & 0.498818 & 0.604938 & 0.779621 & $(0.5836,0.7084)$ \\
\hline 120 & 0.722749 & 0.731771 & 0.818182 & 0.753056 & 0.68171 & 0.664198 & 0.671395 & 0.634845 & 0.55774 & 0.55792 & 0.683951 & 0.687204 & $(0.6187,0.7415)$ \\
\hline 160 & 0.691943 & 0.684896 & 0.732057 & 0.753056 & 0.807601 & 0.775309 & 0.744681 & 0.656325 & 0.707617 & 0.583924 & 0.639506 & 0.682464 & $(0.6447,0.7652)$ \\
\hline 200 & 0.691943 & 0.677083 & 0.739234 & 0.726161 & 0.764846 & 0.77284 & 0.763593 & 0.756563 & 0.749386 & 0.643026 & 0.703704 & 0.744076 & $(0.6688,0.7870)$ \\
\hline 240 & 0.680095 & 0.721354 & 0.741627 & 0.721271 & 0.724466 & 0.728395 & 0.775414 & 0.763723 & 0.781327 & 0.754137 & 0.735802 & 0.739336 & $(0.6806,0.7974)$ \\
\hline
\end{tabular}

UKX
\begin{tabular}{|r|c|c|c|c|c|c|c|c|c|c|c|c|c|}
\hline Moving-window size & Jan & Feb & Mar & Apr & May & Jun & Jul & Aug & Sep & Oct & Nov & Dec & $95 \%$ confidence interval \\
\hline 40 & 0.734957 & 0.622396 & 0.566986 & 0.611247 & 0.648456 & 0.417284 & 0.49409 & 0.50358 & 0.560197 & 0.550827 & 0.701235 & 0.675355 & $(0.5261,0.6550)$ \\
\hline 80 & 0.800948 & 0.708333 & 0.636364 & 0.660147 & 0.653207 & 0.488889 & 0.48227 & 0.393795 & 0.523342 & 0.524823 & 0.582716 & 0.708531 & $(0.5330,0.6602)$ \\
\hline 120 & 0.684834 & 0.697917 & 0.789474 & 0.696822 & 0.667458 & 0.535802 & 0.56974 & 0.441527 & 0.501229 & 0.546099 & 0.575309 & 0.585308 & $(0.5435,0.6711)$ \\
\hline 160 & 0.625592 & 0.601563 & 0.69378 & 0.731051 & 0.750594 & 0.664198 & 0.635934 & 0.539379 & 0.619165 & 0.543735 & 0.54231 & 0.561611 & $(0.5620,0.6897)$ \\
\hline 200 & 0.616114 & 0.588542 & 0.691388 & 0.643032 & 0.68171 & 0.733333 & 0.737589 & 0.615752 & 0.710074 & 0.619385 & 0.624691 & 0.590047 & $(0.5915,0.7175)$ \\
\hline 240 & 0.691943 & 0.632813 & 0.602871 & 0.606357 & 0.629454 & 0.65679 & 0.70922 & 0.687351 & 0.712531 & 0.661939 & 0.698765 & 0.680095 & $(0.6016,0.7271)$ \\
\hline
\end{tabular}

CAC
\begin{tabular}{|r|c|c|c|c|c|c|c|c|c|c|c|c|c|}
\hline Moving-window size & Jan & Feb & Mar & Apr & May & Jun & Jul & Aug & Sep & Oct & Nov & Dec & $95 \%$ confidence interval \\
\hline 40 & 0.71564 & 0.661458 & 0.547847 & 0.674817 & 0.700713 & 0.528395 & 0.472813 & 0.436754 & 0.609337 & 0.65974 & 0.592593 & 0.64455 & $(0.5314,0.6602)$ \\
\hline 80 & 0.772512 & 0.690104 & 0.727273 & 0.723716 & 0.643705 & 0.567801 & 0.539007 & 0.431981 & 0.542998 & 0.513002 & 0.538272 & 0.623223 & $(0.5454,0.6728)$ \\
\hline 120 & 0.580569 & 0.705729 & 0.791866 & 0.745721 & 0.67696 & 0.565432 & 0.56947 & 0.520286 & 0.520885 & 0.510638 & 0.57037 & 0.540284 & $(0.5436,0.6714)$ \\
\hline 160 & 0.606635 & 0.619792 & 0.662679 & 0.738386 & 0.724466 & 0.607407 & 0.652482 & 0.596659 & 0.65602 & 0.513002 & 0.516049 & 0.552133 & $(0.5564,0.6844)$ \\
\hline 200 & 0.620853 & 0.627604 & 0.631579 & 0.633252 & 0.648456 & 0.654321 & 0.661939 & 0.625298 & 0.724816 & 0.664303 & 0.62963 & 0.604265 & $(0.5801,0.7075)$ \\
\hline 240 & 0.675355 & 0.669271 & 0.641148 & 0.682152 & 0.662708 & 0.654321 & 0.631206 & 0.665871 & 0.702703 & 0.661939 & 0.711111 & 0.656398 & $(0.6049,0.7303)$ \\
\hline
\end{tabular}

DAX
\begin{tabular}{|r|c|c|c|c|c|c|c|c|c|c|c|c|c|}
\hline Moving-window size & Jan & Feb & Mar & Apr & May & Jun & Jul & Aug & Sep & Oct & Nov & Dec & $95 \%$ confidence interval \\
\hline 40 & 0.781991 & 0.671875 & 0.566986 & 0.633252 & 0.698337 & 0.560494 & 0.548463 & 0.441527 & 0.501229 & 0.567376 & 0.661728 & 0.767773 & $(0.5534,0.6802)$ \\
\hline 80 & 0.78673 & 0.760417 & 0.708134 & 0.689487 & 0.665083 & 0.580247 & 0.602837 & 0.441527 & 0.555283 & 0.508274 & 0.567901 & 0.696682 & $(0.5667,0.6927)$ \\
\hline 120 & 0.618483 & 0.674479 & 0.779904 & 0.787286 & 0.76247 & 0.693827 & 0.652482 & 0.551313 & 0.572482 & 0.543735 & 0.604938 & 0.601896 & $(0.5908,0.7157)$ \\
\hline 160 & 0.64218 & 0.630208 & 0.669856 & 0.738386 & 0.800475 & 0.753086 & 0.763593 & 0.627685 & 0.670762 & 0.586288 & 0.649383 & 0.677725 & $(0.6230,0.7457)$ \\
\hline 200 & 0.663507 & 0.630208 & 0.636364 & 0.628362 & 0.667458 & 0.74321 & 0.808511 & 0.742243 & 0.761671 & 0.609929 & 0.669136 & 0.6833834 & $(0.6261,0.7486)$ \\
\hline 240 & 0.71564 & 0.679688 & 0.636364 & 0.628362 & 0.631829 & 0.634568 & 0.671395 & 0.701671 & 0.771499 & 0.728132 & 0.767901 & 0.729858 & $(0.6302,0.7526)$ \\
\hline
\end{tabular}


Table 4 (continued)

ELHK

\begin{tabular}{|r|c|c|c|c|c|c|c|c|c|c|c|c|c|}
\hline Moving-window size & Jan & Feb & Mar & Apr & May & Jun & Jul & Aug & Sep & Oct & Nov & Dec & $95 \%$ confidence interval \\
\hline 40 & 0.623223 & 0.434896 & 0.42823 & 0.422893 & 0.567696 & 0.464198 & 0.572104 & 0.625298 & 0.601966 & 0.491276 & 0.555556 & 0.587678 & $(0.4665,0.5980)$ \\
\hline 80 & 0.654028 & 0.598958 & 0.514354 & 0.498778 & 0.432304 & 0.328395 & 0.423168 & 0.52506 & 0.638821 & 0.659574 & 0.614185 & 0.57346 & $(0.4732,0.6034)$ \\
\hline 120 & 0.661137 & 0.596354 & 0.590909 & 0.628362 & 0.574822 & 0.496296 & 0.44208 & 0.508353 & 0.621622 & 0.55792 & 0.683951 & 0.691943 & $(0.5227,0.6524)$ \\
\hline 160 & 0.675355 & 0.664063 & 0.595694 & 0.545232 & 0.586698 & 0.501235 & 0.50591 & 0.584726 & 0.609337 & 0.598109 & 0.609877 & 0.64455 & $(0.5281,0.6583)$ \\
\hline 200 & 0.594787 & 0.627604 & 0.655502 & 0.613692 & 0.586698 & 0.503704 & 0.503546 & 0.677566 & 0.641278 & 0.617201 & 0.604938 & 0.609005 & $(0.5292,0.6596)$ \\
\hline 240 & 0.585308 & 0.606771 & 0.564593 & 0.662592 & 0.634204 & 0.577778 & 0.586288 & 0.661098 & 0.673219 & 0.655118 & 0.597531 & 0.623223 & $(0.5540,0.6832)$ \\
\hline
\end{tabular}

ELIP

\begin{tabular}{|r|c|c|c|c|c|c|c|c|c|c|c|c|l|}
\hline Moving-window size & Jan & Feb & Mar & Apr & May & Jun & Jul & Aug & Sep & Oct & Nov & Dec & $95 \%$ confidence interval \\
\hline 40 & 0.518957 & 0.567708 & 0.607656 & 0.655257 & 0.56057 & 0.446914 & 0.56974 & 0.408115 & 0.457002 & 0.591017 & 0.471605 & 0.379147 & $(0.4537,0.5850)$ \\
\hline 80 & 0.462085 & 0.447917 & 0.555024 & 0.625917 & 0.657957 & 0.590123 & 0.472813 & 0.391408 & 0.400491 & 0.399527 & 0.474074 & 0.409953 & $(0.4250,0.5561)$ \\
\hline 120 & 0.417062 & 0.447917 & 0.502392 & 0.562347 & 0.591449 & 0.595062 & 0.664303 & 0.584726 & 0.422604 & 0.444444 & 0.454321 & 0.390995 & $(0.4412,0.5725)$ \\
\hline 160 & 0.438389 & 0.390625 & 0.488038 & 0.547677 & 0.532067 & 0.528395 & 0.643026 & 0.656325 & 0.641278 & 0.591017 & 0.387654 & 0.402844 & $(0.4562,0.5869)$ \\
\hline 200 & 0.407583 & 0.403646 & 0.476077 & 0.420538 & 0.551069 & 0.575309 & 0.550827 & 0.622912 & 0.579853 & 0.643026 & 0.664198 & 0.516588 & $(0.4694,0.6004)$ \\
\hline 240 & 0.526066 & 0.502604 & 0.437799 & 0.420538 & 0.432304 & 0.45679 & 0.605201 & 0.582339 & 0.5086 & 0.612293 & 0.562963 & 0.521327 & $(0.4485,0.5806)$ \\
\hline
\end{tabular}

UNUS

\begin{tabular}{|r|c|c|c|c|c|c|c|c|c|c|c|c|c|}
\hline Moving-window size & Jan & Feb & Mar & Apr & May & Jun & Jul & Aug & Sep & Oct & Nov & Dec & $95 \%$ confidence interval \\
\hline 40 & 0.751185 & 0.619792 & 0.619617 & 0.606357 & 0.643705 & 0.560494 & 0.583924 & 0.608592 & 0.547912 & 0.498818 & 0.491358 & 0.646919 & $(0.5339,0.6633)$ \\
\hline 80 & 0.689573 & 0.6875 & 0.69378 & 0.731051 & 0.712589 & 0.708642 & 0.63357 & 0.591885 & 0.570025 & 0.510638 & 0.437037 & 0.563981 & $(0.5639,0.6906)$ \\
\hline 120 & 0.604265 & 0.690104 & 0.720096 & 0.784841 & 0.793349 & 0.809877 & 0.732861 & 0.615752 & 0.621622 & 0.534279 & 0.57037 & 0.540284 & $(0.6061,0.7291)$ \\
\hline 160 & 0.597156 & 0.596354 & 0.684211 & 0.743276 & 0.800475 & 0.807407 & 0.718676 & 0.651551 & 0.63145 & 0.598109 & 0.590123 & 0.590047 & $(0.6057,0.7295)$ \\
\hline 200 & 0.632701 & 0.627604 & 0.681818 & 0.694377 & 0.774347 & 0.797531 & 0.758865 & 0.71599 & 0.668305 & 0.588652 & 0.587654 & 0.630332 & $(0.6186,0.7416)$ \\
\hline 240 & 0.64218 & 0.679688 & 0.665072 & 0.652812 & 0.726841 & 0.698765 & 0.758865 & 0.661098 & 0.685504 & 0.676123 & 0.641975 & 0.611374 & $(0.6129,0.7373)$ \\
\hline
\end{tabular}

ELUK

\begin{tabular}{|r|c|c|c|c|c|c|c|c|c|c|c|c|c|}
\hline Moving-window size & Jan & Feb & Mar & Apr & May & Jun & Jul & Aug & Sep & Oct & Nov & Dec & $95 \%$ confidence interval \\
\hline 40 & 0.654208 & 0.744792 & 0.61244 & 0.564792 & 0.665083 & 0.483951 & 0.475177 & 0.610979 & 0.624079 & 0.468085 & 0.560494 & 0.443128 & $(0.5096,0.6393)$ \\
\hline 80 & 0.611374 & 0.622396 & 0.696172 & 0.669927 & 0.705643 & 0.602469 & 0.659574 & 0.599045 & 0.545455 & 0.58156 & 0.6 & 0.537915 & $(0.5551,0.6837)$ \\
\hline 120 & 0.580569 & 0.539063 & 0.624402 & 0.640587 & 0.724466 & 0.745679 & 0.624113 & 0.565632 & 0.614251 & 0.567376 & 0.585185 & 0.632701 & $(0.5564,0.6848)$ \\
\hline 160 & 0.606635 & 0.59375 & 0.617225 & 0.618582 & 0.693587 & 0.703704 & 0.72104 & 0.673031 & 0.636364 & 0.605201 & 0.585185 & 0.56872 & $(0.5717,0.6994)$ \\
\hline 200 & 0.618483 & 0.627604 & 0.62201 & 0.682152 & 0.662708 & 0.683951 & 0.643026 & 0.687351 & 0.638821 & 0.591017 & 0.632099 & 0.623223 & $(0.5788,0.7064)$ \\
\hline 240 & 0.632701 & 0.648438 & 0.629187 & 0.643032 & 0.686461 & 0.664198 & 0.567376 & 0.620525 & 0.577396 & 0.555556 & 0.597531 & 0.587678 & $(0.5526,0.6818)$ \\
\hline
\end{tabular}

EPFR

\begin{tabular}{|r|c|c|c|c|c|c|c|c|c|c|c|c|l|}
\hline Moving-window size & Jan & Feb & Mar & Apr & May & Jun & Jul & Aug & Sep & Oct & Nov & Dec & $95 \%$ confidence interval \\
\hline 40 & 0.684834 & 0.705729 & 0.748804 & 0.630807 & 0.63658 & 0.530864 & 0.48227 & 0.508353 & 0.589681 & 0.567376 & 0.577778 & 0.533175 & $(0.5346,0.6635)$ \\
\hline 80 & 0.689573 & 0.666667 & 0.727273 & 0.814181 & 0.793349 & 0.587654 & 0.539007 & 0.52506 & 0.503686 & 0.531915 & 0.57037 & 0.57346 & $(0.5637,0.6896)$ \\
\hline 120 & 0.687204 & 0.721354 & 0.803828 & 0.767726 & 0.80285 & 0.720988 & 0.704492 & 0.613365 & 0.53317 & 0.465721 & 0.516049 & 0.597156 & $(0.5996,0.7223)$ \\
\hline 160 & 0.575829 & 0.690104 & 0.717703 & 0.740831 & 0.760095 & 0.738272 & 0.742317 & 0.680191 & 0.65602 & 0.588652 & 0.62963 & 0.578199 & $(0.6126,0.7363)$ \\
\hline 200 & 0.632701 & 0.614583 & 0.650718 & 0.669927 & 0.738717 & 0.745679 & 0.754137 & 0.73031 & 0.72973 & 0.680851 & 0.706173 & 0.689573 & $(0.6346,0.7567)$ \\
\hline 240 & 0.748815 & 0.710938 & 0.712919 & 0.669927 & 0.738717 & 0.792593 & 0.808511 & 0.775656 & 0.808354 & 0.711584 & 0.775309 & 0.746445 & $(0.6927,0.8075)$ \\
\hline
\end{tabular}

EPGR

\begin{tabular}{|r|c|c|c|c|c|c|c|c|c|c|c|c|c|}
\hline Moving-window size & Jan & Feb & Mar & Apr & May & Jun & Jul & Aug & Sep & Oct & Nov & Dec & $95 \%$ confidence interval \\
\hline 40 & 0.5 & 0.619792 & 0.509569 & 0.479218 & 0.674584 & 0.528395 & 0.397163 & 0.494033 & 0.542998 & 0.437352 & 0.530864 & 0.4333649 & $(0.4454,0.5772)$ \\
\hline 80 & 0.471564 & 0.434896 & 0.617225 & 0.586797 & 0.660333 & 0.558025 & 0.486998 & 0.498807 & 0.402948 & 0.460993 & 0.520998 & 0.447867 & $(0.4468,0.5786)$ \\
\hline 120 & 0.485782 & 0.466146 & 0.550239 & 0.535452 & 0.67696 & 0.582716 & 0.527187 & 0.539379 & 0.501229 & 0.491726 & 0.501235 & 0.549763 & $(0.4684,0.6006)$ \\
\hline 160 & 0.471564 & 0.510417 & 0.590909 & 0.515892 & 0.603325 & 0.562963 & 0.555556 & 0.508353 & 0.513514 & 0.588652 & 0.595062 & 0.521327 & $(0.4788,0.6111)$ \\
\hline 200 & 0.511848 & 0.453125 & 0.535885 & 0.572127 & 0.586698 & 0.479012 & 0.463357 & 0.505967 & 0.484029 & 0.510638 & 0.548148 & 0.552133 & $(0.4510,0.5837)$ \\
\hline 240 & 0.469194 & 0.552083 & 0.638756 & 0.520782 & 0.555819 & 0.54321 & 0.49401 & 0.431981 & 0.402948 & 0.527187 & 0.518519 & 0.495261 & $(0.4461,0.5785)$ \\
\hline
\end{tabular}

